\title{
乌ु
}

\section{X-ray Detection of Transient Magnetic Moments Induced by a Spin Current in $\mathrm{Cu}$}

\author{
R. Kukreja, ${ }^{1,2, *}$ S. Bonetti, ${ }^{1,3}$ Z. Chen, ${ }^{1,3}$ D. Backes, ${ }^{4}$ Y. Acremann, ${ }^{5}$ J. A. Katine, ${ }^{6}$ \\ A. D. Kent, ${ }^{4}$ H. A. Dürr, ${ }^{1}$ H. Ohldag, ${ }^{7}$ and J. Stöhr ${ }^{1, \dagger}$ \\ ${ }^{1}$ Stanford Institute for Materials and Energy Sciences, SLAC National Accelerator Laboratory, \\ 2575 Sand Hill Road, Menlo Park, California 94025, USA \\ ${ }^{2}$ Department of Materials Science and Engineering, Stanford University, 496 Lomita Mall, Stanford, California 94305, USA \\ ${ }^{3}$ Department of Physics, Stanford University, 382 Via Pueblo Mall, Stanford, California 94305, USA \\ ${ }^{4}$ Physics Department, New York University, 4 Washington Place, New York, New York 10003, USA \\ ${ }^{5}$ Laboratorium für Festkörperphysik, ETH Zürich, HPF C 5, Otto-Stern-Weg 1, Zürich 8093, Switzerland \\ ${ }^{6}$ HGST, a Western Digital Company, 3403 Yerba Buena Road, San Jose, California 95135, USA \\ ${ }^{7}$ Stanford Synchrotron Radiation Lightsource, SLAC National Accelerator Laboratory, \\ 2575 Sand Hill Road, Menlo Park, California 94025, USA
}

(Received 9 March 2015; revised manuscript received 2 June 2015; published 24 August 2015)

\begin{abstract}
We have used a MHz lock-in x-ray spectromicroscopy technique to directly detect changes in magnetic moment of $\mathrm{Cu}$ due to spin injection from an adjacent $\mathrm{Co}$ layer. The elemental and chemical specificity of $\mathrm{x}$ rays allows us to distinguish two spin current induced effects. We detect the creation of transient magnetic moments of $3 \times 10^{-5} \mu_{B}$ on $\mathrm{Cu}$ atoms within the bulk of the $28 \mathrm{~nm}$ thick $\mathrm{Cu}$ film due to spin accumulation. The moment value is compared to predictions by Mott's two current model. We also observe that the hybridization induced existing magnetic moments at the $\mathrm{Cu}$ interface atoms are transiently increased by about $10 \%$ or $4 \times 10^{-3} \mu_{B}$ per atom. This reveals the dominance of spin-torque alignment over Joule heat induced disorder of the interfacial $\mathrm{Cu}$ moments during current flow.
\end{abstract}

DOI: 10.1103/PhysRevLett.115.096601

PACS numbers: 72.25.Mk, 72.15.-v, 78.47.db

One of the new paradigms in magnetism research is the use of spin currents to read and write static magnetic bits via the giant magnetoresistance [1] and spin transfer torque effects $[2,3]$. Spin currents are also believed to play a key role in the ultrafast manipulation of the magnetization by femtosecond optical pulses, like in all optical switching [4-6]. They exist even during current flow through nonmagnetic materials consisting of atoms with large spin-orbit coupling such as Pt, leading to spin accumulation through the spin Hall or Rashba effects $[7,8]$. The presence of spin currents is typically revealed through current or voltage dependent measurements, but an atomic level understanding of the detailed spin dependent scattering mechanisms requires techniques that can directly probe the electronic structure at the nanoscale.

In this Letter, we report the direct detection of transient magnetization in a nonmagnet $(\mathrm{Cu})$ caused by injection of spin polarized current from an adjacent ferromagnet (Co). This is accomplished through significant advancement in the sensitivity of scanning transmission microscope (STXM) which is achieved by using time dependent modulation of a spin current synchronized with $\mathrm{x}$-ray pulses to produce a sensitivity corresponding to the magnetic moment of about $50 \mathrm{Fe}$ atoms. Using this technique, we were able to measure an extremely small transient $\mathrm{x}$-ray magnetic circular dichroism (XMCD) effect in the nonmagnetic $\mathrm{Cu}$ layer.

Our experimental arrangement, shown in Fig. 1, was similar to that employed in previous studies of spin-torque switching [9]. However, instead of observing directional changes of the large atomic magnetic moments of $\simeq 2 \mu_{B}$ in a ferromagnetic Co layer, we used the XMCD effect to quantitatively measure the tiny, $\simeq 10^{-5} \mu_{B}$ /atom, transient signal due to spin currents in interfacial and bulk $\mathrm{Cu}$ atoms.

The samples consisted of multilayer structure grown by multilayer grown by sputtering, where the ferromagnetic polarizer layer $[0.3 \mathrm{Co} / 0.9 \mathrm{Pd}]_{6} / 0.3 \mathrm{Co} /[0.6 \mathrm{Ni} / 0.09 \mathrm{Co}]_{3} /$ $0.21 \mathrm{Co}$ (all the dimensions are in $\mathrm{nm}$ ) was designed to have a strong perpendicular magnetic anisotropy and large spin polarization, as previously discussed in [10]. It is important to note that the Co and Ni layers were deposited at room temperature where they are immiscible so that the resulting $\mathrm{Co} / \mathrm{Ni}$ multilayer exhibits perpendicular anisotropy and the final interface consists of $\mathrm{Co} / \mathrm{Cu}$. The out-of-plane magnetization for the ferromagnetic layer facilitated XMCD imaging in the transmission geometry. The ferromagnetic Co interface layer was followed by a $\mathrm{Cu}$ layer of $28 \mathrm{~nm}$. The multilayer stack was fabricated into a nanopillar by using electron beam lithography with a top contact of $5 \mathrm{Cr} / 50 \mathrm{Au}$ and a bottom contact of $3 \mathrm{Ta} / 30 \mathrm{Ru} / 3 \mathrm{Ta} / 30 \mathrm{Ru} / 5 \mathrm{Ta} / 2 \mathrm{Pd}$. The pillar had a resistance of $\sim 47 \Omega$. In order to measure the $\mathrm{x}$-ray transmission through the pillar, the Si wafer on the backside was etched leaving the sample supported by a $\operatorname{SiN}_{x}$ membrane. The key parts of the sample structure are shown in Fig. 1(c).

The measurements were performed at the STXM beamline (13-1) at the Stanford Synchrotron Radiation Lightsource shown in Fig. 1(b). Circularly polarized x rays from an undulator were focused to a spot size of $35 \mathrm{~nm}$ by a 
(a)

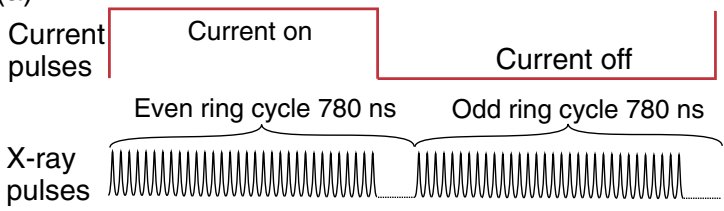

(b)

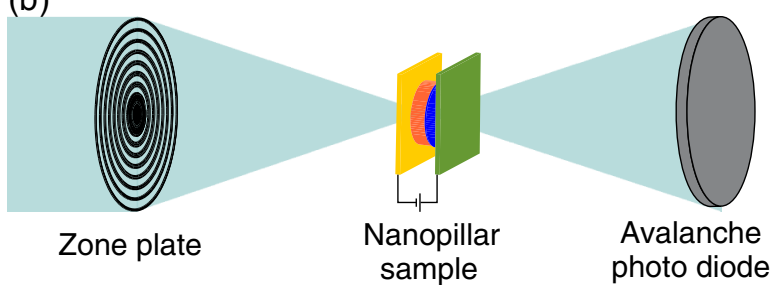

(c) current $+5 \mathrm{~mA}$
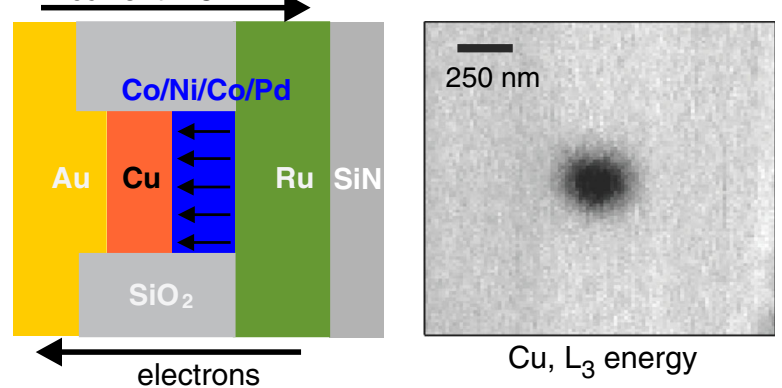

FIG. 1 (color online). (a) Timing of current pulses, x-ray pulses, and data collection periods, as discussed in the text. (b) Schematic of the X-ray microscopy measurements. The X-ray spot size at the sample was $35 \mathrm{~nm}$ and the transmitted $\mathrm{x}$ rays were detected by an avalanche photo diode. Images were recorded by raster scanning of the sample. (c) The sample consisted of a nanopillar of $240 \mathrm{~nm}$ diameter containing a ferromagnetic multilayer with perpendicular magnetization direction, as discussed in the text. Current to the pillar was supplied by $\mathrm{Au}$ and $\mathrm{Ru}$ contact leads, as shown. The current is defined as positive when flowing from $\mathrm{Cu}$ to the ferromagnet, corresponding to electron flow in the opposite direction. At the bottom right, we show a representative STXM contrast image revealing the nanopillar, taken at the $\mathrm{Cu} L_{3}$ resonance energy of $932.7 \mathrm{eV}$.

zone plate lens and the transmitted intensity was measured with an avalanche photo diode. The sample was raster scanned to generate an image. Current-on or -off periods applied to the sample were synchronized with the cycle time of the storage ring (781.2 ns) using the counting electronics [11], as illustrated in Fig. 1(a). This detection scheme [12] allowed us to reach a sensitivity of $1 \times 10^{-5}$, more than an order of magnitude increase in sensitivity over previous attempts $\left(6 \times 10^{-4}\right)$ to detect spin accumulation with $\mathrm{x}$ rays [13].

Figure 2(a) shows the averaged line scan across the nanopillar, for the transmitted current-on or -off intensity ratio, $I^{\sigma \pm}=I_{\text {on }}^{\sigma \pm} / I_{\text {off }}^{\sigma \pm}$, recorded with plus $(\sigma+$, blue $)$ and minus $(\sigma-$, red) $\mathrm{x}$-ray helicities. This measurement was taken at $-5 \mathrm{~mA}$, which corresponds to a current density of $10^{11} \mathrm{~A} / \mathrm{m}^{2}$. Figure 2(b) shows similar line scans for the opposite direction of current flow $(+5 \mathrm{~mA})$. The inset shows current dependence of the XMCD contrast, defined

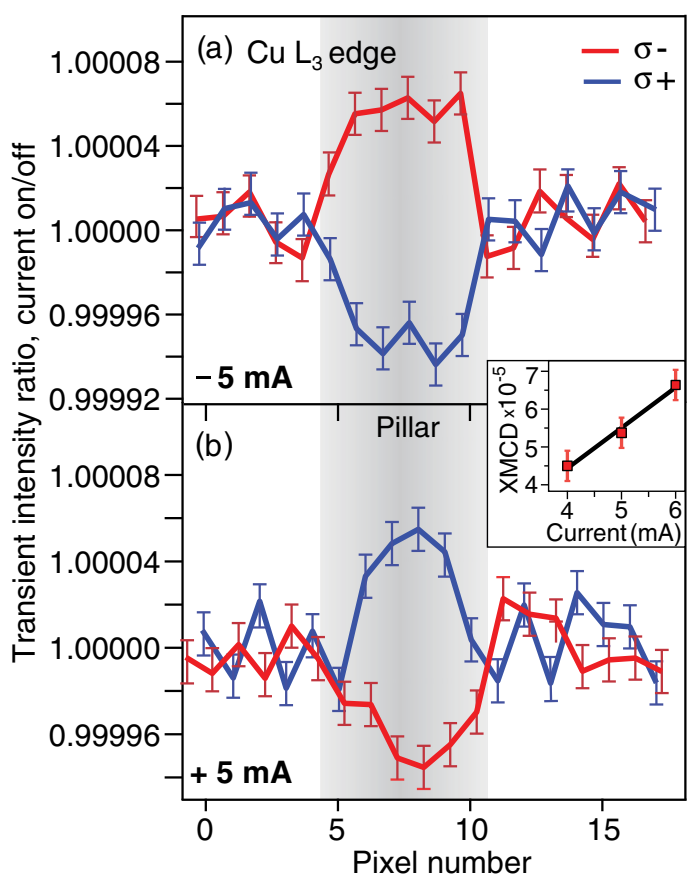

FIG. 2 (color online). (a) Line scan across the nanopillar. Plotted is the ratio of the transmitted intensities for current on versus off, $I^{\sigma \pm}=I_{\text {on }}^{\sigma \pm} / I_{\text {off }}^{\sigma \pm}$, recorded for minus $\left(\sigma^{-}\right)$and plus $\left(\sigma^{+}\right)$helicities of the incident $x$ rays and $-5 \mathrm{~mA}$ current. (b) Same as in (a) for opposite current direction $(+5 \mathrm{~mA})$. The inset shows the current dependence of the XMCD contrast, defined as the intensity ratio $\left(I^{\sigma+}-I^{\sigma-}\right) /\left(I^{\sigma+}+I^{\sigma-}\right)$.

as $\left(I^{\sigma+}-I^{\sigma-}\right) /\left(I^{\sigma+}+I^{\sigma-}\right)$, which increases linearly with the current. The current-on or -off normalization removes any contrast due to topography and static magnetization. The dependence of image contrast on $\mathrm{x}$-ray polarization and current flow direction in Figs. 2(a) and 2(b) demonstrates

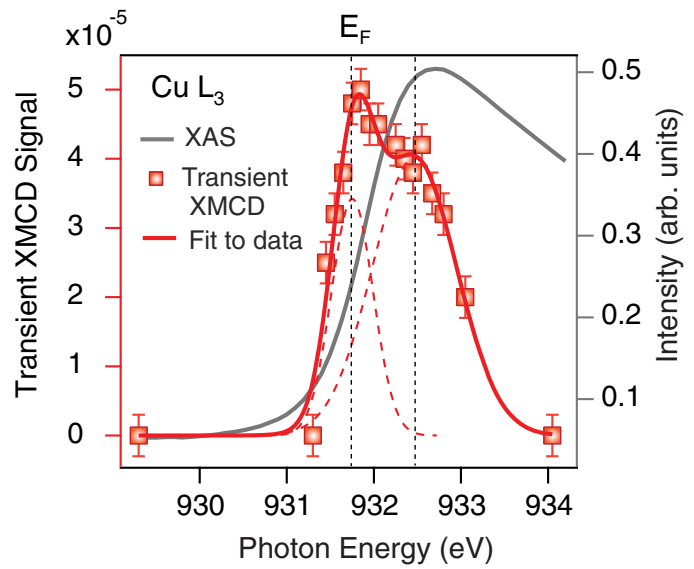

FIG. 3 (color online). Comparison of the $L_{3} \mathrm{X}$-ray absorption spectrum (XAS) of $\mathrm{Cu}$ metal (gray line) with the transient $\mathrm{Cu} \mathrm{XMCD}$ signal (red squares) of the nanopillar sample, recorded with $+5 \mathrm{~mA}$ current-on or -off. A fit of the transient spectrum (red line) composed of two Gaussians (dashed red) is shown superimposed. 
that this contrast is magnetic. The size of the contrast is similar for both current directions within an error margin of $1 \times 10^{-5}$.

Figure 3 shows the transient XMCD spectrum (red data points) obtained from integrated image intensities as a function of photon energy across the $\mathrm{Cu} L_{3}$ resonance with an applied current of $+5 \mathrm{~mA}$. The transient $\mathrm{Cu}$ XMCD signal exhibits a two peak structure, clearly revealed by Gaussian fits (dashed red curves). The lower peak is centered at the inflection point of the $\mathrm{Cu}$ metal $\mathrm{x}$-ray absorption spectrum (XAS, gray curve), and has a full width at half maximum of $0.57 \mathrm{eV}$. The second peak is located $0.7 \mathrm{eV}$ higher in energy and has a full width at half maximum of $1.0 \mathrm{eV}$.

We assign the lower energy transient XMCD peak in Fig. 3 to $\mathrm{Cu}$ atoms in the bulk of the $28 \mathrm{~nm}$ thick film. It is due to spin accumulation induced by a mismatch of the spin dependent resistivities across a $\mathrm{Co} / \mathrm{Cu}$ interface and exists over a distance that is determined by the spin diffusion length [14]. For our sample, the spin accumulation is approximately constant across the $\mathrm{Cu}$ layer whose thickness of $28 \mathrm{~nm}$ is much smaller than the $\mathrm{Cu}$ spin diffusion length of $\simeq 350 \mathrm{~nm}[15,16]$. Our assignment is supported by the fact that its position coincides with the inflection point of the $\mathrm{Cu}$ metal XAS spectrum which corresponds to the position of the Fermi level $E_{F}$ [17]. The XMCD peak also has the minimum width allowed by the $2 p_{3 / 2}$ core hole lifetime $(\simeq 0.5 \mathrm{eV})[17,18]$, which is in good accord with the notion that within the bulk of the $\mathrm{Cu}$ film the transient spins flow and accumulate within a narrow energy band around the Fermi level.

The higher energy peak position in Fig. 3 is close to the peak of the XAS spectrum. Based on earlier work on $\mathrm{Co} / \mathrm{Cu}$ multilayers $[19,20]$ and detailed studies of the $\mathrm{XMCD}$ and XAS spectra of $\mathrm{Co} / \mathrm{Cu}$ alloys, it is assigned to magnetic $\mathrm{Cu}$ interface atoms with a room temperature moment of $\simeq 0.05 \mu_{B}[19,20]$. For our sample with a single $\mathrm{Co} / \mathrm{Cu}$ interface, the static XMCD peak of the interface atoms was too weak to be detected since the $\mathrm{Cu}$ signal is dominated by the bulk of the film. However, its transient XMCD signal was observable owing to the high sensitivity of our $\mathrm{MHz}$ lock-in current-on or -off technique.

For the same ferromagnetic alignment direction of the $\mathrm{Co}$ moments in multilayers and $\mathrm{Co} / \mathrm{Cu}$ alloy reference samples and in our pillar sample, we find that the signs of the static $\mathrm{Co}$ and $\mathrm{Cu}$ interface XMCD peaks and those of the two transient peaks in Fig. 3 are the same for a $+5 \mathrm{~mA}$ current direction. From the size of the integrated transient $\mathrm{Cu}$ XMCD signal we can estimate the magnetic moment per $\mathrm{Cu}$ atom due to the spin currents using the procedure in Ref. [19]. Exploiting the fact that the density of states for pure $\mathrm{Cu}$ [17] and a $\mathrm{Cu}$ layer sandwiched between $\mathrm{Co}$ [21] exhibits more $d$ than $s$ states around $E_{F}$ and that the XMCD signal is dominated by $2 p_{3 / 2} \rightarrow 3 d$ transitions, we derive a moment of $m_{\mathrm{Cu}} \simeq 3 \times 10^{-5} \mu_{B}$ per $\mathrm{Cu}$ atom for the lower energy peak. If we assign the intensity of the second peak to a single layer of $\mathrm{Cu}$ interface atoms, the transient moment per atom is $m_{\mathrm{Cu}} \simeq 4 \times 10^{-3} \mu_{B}$.

The size and sign of the transient $\mathrm{Cu}$ moment of the lower energy XMCD peak in Fig. 3, which is assigned to spin accumulation in the bulk of the $28 \mathrm{~nm}$ thick $\mathrm{Cu}$ film, can both be explained by Mott's two current model [22,23]. In this model the current flows in independent, parallel spin-up and spin-down channels, and spin-flip scattering is forbidden. In each spin channel, the resistivity is determined by scattering of itinerant $s-p$ electrons into empty $d$ states localized on the atomic sites. In a strong ferromagnet like Co, the majority $d$ states lie below the Fermi energy and are filled. The resistivity is therefore determined by transitions of minority $s$ - $p$ electrons into minority $d$ holes in accordance with the band structure schematically illustrated in Fig. 4(a). In $\mathrm{Cu}$, the $d$ band lies well below the Fermi level and the lack of localized empty $d$ states in both spin channels leads to a low resistivity.

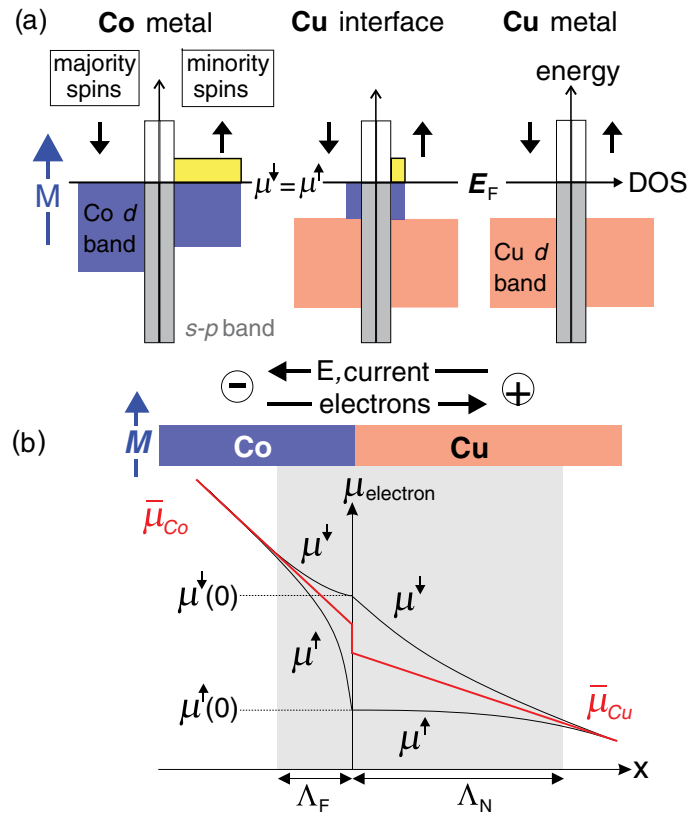

FIG. 4 (color online). (a) Schematic density of states (DOS) without current flow $\left(E_{F}=\mu^{\uparrow}=\mu^{\downarrow}\right)$ for itinerant $s$ - $p$ and localized $d$ spins for bulk $\mathrm{Co}$ and $\mathrm{Cu}$, and $\mathrm{Cu}$ interface atoms. The exchange-split $d$ DOS of the Co and magnetic $\mathrm{Cu}$ interface atoms exhibits occupied (blue) and unoccupied minority spin states (yellow), which is absent in bulk $\mathrm{Cu}$. The static XMCD effect arises from transitions to the yellow shaded unoccupied $d$ states. (b) Model of the spin dependent electron chemical potentials in the presence of electron spin flow from $\mathrm{Co}$ to $\mathrm{Cu}$ across an abrupt interface without interface states. The diagram corresponds to the shown Co magnetization direction and $+5 \mathrm{~mA}$ current as in Fig. 1(c). The spin averaged chemical potentials $\bar{\mu}_{\mathrm{Co}}$ in $\mathrm{Co}$ and $\bar{\mu}_{\mathrm{Cu}}$ in $\mathrm{Cu}$ are shown in red. The chemical potentials decay exponentially with distance from their maximum values $\mu^{\uparrow}(0)$ and $\mu^{\downarrow}(0)$ at the interface. The origin of the transient $\mathrm{XMCD}$ effect is discussed in the text. 
When electrons flow from $\mathrm{Co}$ to $\mathrm{Cu}$, the minority spins experience a lower resistance in $\mathrm{Cu}$ since there are less empty $d$ states to scatter into. The Co side of the interface region is therefore preferentially depleted of minority spins. Charge neutrality then requires accumulation of the majority spins. Since there must be continuity in each spin channel across the interface, the accumulation of majority spins exists on both sides of the interface, as illustrated in Fig. 4(b). For electrons flowing from $\mathrm{Cu}$ to $\mathrm{Co}$, the minority spins experience a higher resistance when entering Co. This leads to accumulation of minority spins near the interface.

The sign and magnitude of the transient $\mathrm{Cu}$ XMCD intensity can be estimated by assuming a change of the spin dependent resistivities and chemical potentials at an interface between two bulklike layers [24]. The magnetic moment due to spin accumulation near a ferromagnet $(F)$ and nonmagnet $(N)$ interface is derived in Ref. [3] and for $\ell=F$ or $N$ is given by

$$
\begin{aligned}
m_{\ell} & =-D_{\ell}\left(E_{F}\right)\left[\mu^{\uparrow}(0)-\mu^{\downarrow}(0)\right] \frac{1}{d} \int_{0}^{d} e^{-x / \Lambda_{\ell}} d x \mu_{B} \\
& =D_{\ell}\left(E_{F}\right) \frac{2\left(2 \alpha_{F}-1\right) \Lambda_{N} \rho_{N} e j}{1+4 \alpha_{F}\left(1-\alpha_{F}\right) \frac{\Lambda_{N} \rho_{N}}{\Lambda_{F} \rho_{F}}} \frac{\Lambda_{\ell}}{d}\left[1-e^{-d / \Lambda_{\ell}}\right] \mu_{B} .
\end{aligned}
$$

We can estimate the transient $\mathrm{Cu}$ moment for our layer thickness $d_{\mathrm{Cu}}=28 \mathrm{~nm}$. We assume that the spin current is due to the final Co layer from which electrons flow into $\mathrm{Cu}$ and use the following values for the spin dependent parameters for bulk $\mathrm{Cu}$ and $\mathrm{Co}$ [15]: the densities of states at the Fermi level $D_{\mathrm{Co}}\left(E_{F}\right)=0.8$ atom $^{-1} \mathrm{eV}^{-1}$ and $D_{\mathrm{Cu}}\left(E_{F}\right)=0.2$ atom $^{-1} \mathrm{eV}^{-1}$, the spin asymmetry parameter for Co conduction $\alpha_{\mathrm{Co}}=0.8$, the spin diffusion lengths $\Lambda_{\mathrm{Co}}=38 \mathrm{~nm}$ and $\Lambda_{\mathrm{Cu}}=350 \mathrm{~nm}$, and the resistivities $\rho_{\mathrm{Co}}=210 \Omega \mathrm{nm}$ and $\rho_{\mathrm{Cu}}=17 \Omega \mathrm{nm}$. For $j=+1 \times 10^{11} \mathrm{~A} / \mathrm{m}^{2}$, we obtain the transient $\mathrm{Cu}$ moments to be $m_{\mathrm{Cu}} \simeq 9.3 \times 10^{-5} \mu_{B}$, a factor of 3 larger than our experimental value of $3 \times 10^{-5} \mu_{B}$.

Our model explains the sign reversals with the direction of current flow shown in Fig. 2 and accounts for the fact that the moment due to spin accumulation on "bulk" $\mathrm{Cu}$ atoms revealed by Fig. 3 has the same sign (direction) as the static moments in the previously measured alloys [19]. For the Co magnetization direction in Fig. 4(a), the directions of the static $\mathrm{Co}$ and $\mathrm{Cu}$ interface moments are parallel, since in both cases there are more minority $d$ holes, identified by yellow shading. The sign of the spin accumulation moment in the bulk of the $\mathrm{Cu}$ film follows from Fig. 4(b), which reveals a lowering of the chemical potential for the minority spins resulting in a surplus of minority holes. Hence, in all cases the moment direction is the same.

The higher energy peak in Fig. 3 cannot be explained within the above model which ignores the existence of interface states. Our results reveal a current induced transient increase of $m_{\mathrm{Cu}} \simeq 4 \times 10^{-3} \mu_{B}$ or about $10 \%$ of the $0.05 \mu_{B}$ static magnetic moment per $\mathrm{Cu}$ interface atom.
Although the two peaks have about the same size in Fig. 3, the relative abundance of bulk and interface $\mathrm{Cu}$ atoms means that the moment change per $\mathrm{Cu}$ atom is about 2 orders of magnitude larger for the interface $\left(4 \times 10^{-3} \mu_{B}\right)$ than the bulk $\left(3 \times 10^{-5} \mu_{B}\right) \mathrm{Cu}$ atoms.

We attribute the large difference in the transient moments to the fact that the interface $\mathrm{Cu}$ atoms themselves are magnetic through direct hybridization of their $d$ states with Co $[19,20]$, yet are more sensitive to thermal and spin current effects than the Co moments. Indeed, the sign of the second peak in Fig. 3 corresponds to a 10\% increase of the $\mathrm{Cu}$ interface moments in the current-on relative to the current-off cycle. Current pulsing leads to an average temperature increase in the pillar of order $100 \mathrm{~K}$ above room temperature. Because of the cooling time scale being longer than the duration of the current-off cycle, the sample remains above room temperature even during the currentoff cycle. While the Co magnetization remains stable within the temperature range $<400 \mathrm{~K}$, the $\mathrm{Cu}$ interface moments are expected to decrease [25]. When the current is on, we do not observe a further decrease of the $\mathrm{Cu}$ interface moments due to Ohmic heating but rather a spin current induced increase. We attribute it to a spin-torque alignment or stabilization of the $\mathrm{Cu}$ interface moments. Finally, we mention that conservation of angular momentum during the interfacial spin-torque process will lower the spin current polarization in the bulk of the $\mathrm{Cu}$ film, in accord with the lower value measured than calculated value.

This work is supported by the Department of Energy, Office of Science, Basic Energy Sciences, Materials Sciences and Engineering Division, under Contract No. DE-AC02-76SF00515. Use of the Stanford Synchrotron Radiation Lightsource, SLAC National Accelerator Laboratory, is supported by the U.S. Department of Energy, Office of Science, Office of Basic Energy Sciences under Contract No. DE-AC0276SF00515. Work done at NYU was supported by NSF-DMR-1309202. S. B. acknowledges support from the Knut and Alice Wallenberg Foundation.

Note added.-After submission of our paper we became aware of related spin-pumping XMCD studies by J. Li et al. [26].

*roopali.kukreja@gmail.com

†stohr@slac.stanford.edu

[1] M. N. Baibich, J. M. Broto, A. Fert, F. Nguyen Van Dau, F. Petroff, P. Etienne, G. Creuzet, A. Friederich, and J. Chazelas, Giant Magnetoresistance of (001)Fe/(001)Cr Magnetic Superlattices, Phys. Rev. Lett. 61, 2472 (1988); G. Binsach, P. Grünberg, F. Saurenbach, and W. Zinn, Enhanced magnetoresistance in layered magnetic structures with antiferromagnetic interlayer exchange, Phys. Rev. B 39, 4828 (1989). 
[2] J. C. Slonczewski, Current-driven excitation of magnetic multilayers, J. Magn. Magn. Mater. 159, L1 (1996); L. Berger, Emission of spin waves by a magnetic multilayer traversed by a current, Phys. Rev. B 54, 9353 (1996).

[3] J. Stöhr and H.C. Siegmann, Magnetism: From Fundamentals to Nanoscale Dynamics (Springer, New York, 2006).

[4] C. D. Stanciu, F. Hansteen, A. V. Kimel, A. Kirilyuk, A. Tsukamoto, A. Itoh, and Th. Rasing, All-Optical Magnetic Recording with Circularly Polarized Light, Phys. Rev. Lett. 99, 047601 (2007).

[5] I. Radu et al., Transient ferromagnetic-like state mediating ultrafast reversal of antiferromagnetically coupled spins, Nature (London) 472, 205 (2011).

[6] C. E. Graves et al., Nanoscale spin reversal by non-local angular momentum transfer following ultrafast laser excitation in ferrimagnetic GdFeCo, Nat. Mater. 12, 293 (2013).

[7] For a review see J. Inoue and H. Ohno, Taking the Hall effect for a spin, Science 309, 2004 (2005).

[8] I. M. Miron et al., Current-driven spin torque induced by the Rashba effect in a ferromagnetic metal layer, Nat. Mater. 9, 230 (2010).

[9] Y. Acremann, J. P. Strachan, V. Chembrolu, S. D. Andrews, T. Tyliszczak, J. A. Katine, M. J. Carey, B. M. Clemens, H. C. Siegmann, and J. Stöhr, Time-Resolved Imaging of Spin Transfer Switching: Beyond the Macrospin Concept, Phys. Rev. Lett. 96, 217202 (2006); J. P. Strachan et al., Direct Observation of Spin-Torque Driven Magnetization Reversal through Nonuniform Modes, Phys. Rev. Lett. 100, 247201 (2008); D. P. Bernstein et al., Nonuniform switching of the perpendicular magnetization in a spin-torquedriven magnetic nanopillar, Phys. Rev. B 83, 180410 (2011).

[10] J.-M. L. Beaujour, W. Chen, K. Krycka, C.-C. Kao, J. Z. Sun, and A. D. Kent, Ferromagnetic resonance study of sputtered ColNi multilayers, Eur. Phys. J. B 59, 475 (2007); M. Gottwald et al., Co/Ni(111) superlattices studied by microscopy, x-ray absorption, and ab initio calculations, Phys. Rev. B 86, 014425 (2012).

[11] Y. Acremann, V. Chembrolu, J. P. Strachan, T. Tyliszczak, and J. Stöhr, Software defined photon counting system for time resolved x-ray experiments, Rev. Sci. Instrum. 78, 014702 (2007).

[12] S. Bonetti et al., Microwave soft x-ray microscopy for nanoscale magnetization dynamics in the $5-10 \mathrm{GHz}$ frequency range, arXiv:1504.07561.
[13] O. Mosendz, G. Mihajlović, J. E. Pearson, P. Fischer, M.-Y. Im, S. D. Bader, and A. Hoffmann, Imaging of lateral spin valves with soft x-ray microscopy, Phys. Rev. B 80, 104439 (2009).

[14] M. Johnson and R.H. Silsbee, Interfacial Charge-Spin Coupling: Injection and Detection of Spin Magnetization in Metals, Phys. Rev. Lett. 55, 1790 (1985).

[15] J. Bass and W. P. Pratt, Spin-diffusion lengths in metals and alloys, and spin-flipping at metal/metal interfaces: an experimentalist's critical review, J. Phys. Condens. Matter 19, 183201 (2007).

[16] F. J. Jedema, T. Filip, and B. J. van Wees, Electrical spin injection and accumulation at room temperature in an allmetal mesoscopic spin valve, Nature (London) 410, 345 (2001).

[17] H. Ebert, J. Stöhr, S. S. P. Parkin, M. Samant, and A. Nilsson, L-edge x-ray absorption in fcc and bec $\mathrm{Cu}$ metal: Comparison of experimental and first-principles theoretical results, Phys. Rev. B 53, 16067 (1996).

[18] J. E. Müller, O. Jepsen, and J. W. Wilkins, X-ray absorption spectra: K-edges of $3 \mathrm{~d}$ transition metals, L-edges of $3 \mathrm{~d}$ and $4 \mathrm{~d}$ metals, and M-edges of palladium, Solid State Commun. 42, 365 (1982).

[19] M. G. Samant et al., Induced Spin Polarization in Cu Spacer Layers in $\mathrm{Co} / \mathrm{Cu}$ Multilayers, Phys. Rev. Lett. 72, 1112 (1994).

[20] R. Wu and A. J. Freeman, Magnetic circular dichroism at the $\mathrm{K}$ and $\mathrm{L}$ edges of $\mathrm{Co}$ and $\mathrm{Cu}$ in $\mathrm{Co} / \mathrm{Cu}(001)$, J. Appl. Phys. 79, 6500 (1996).

[21] A. Nilsson et al., Determination of the electronic density of states near buried interfaces: Application to $\mathrm{Co} / \mathrm{Cu}$ multilayers, Phys. Rev. B 54, 2917 (1996).

[22] N. F. Mott, The electrical conductivity of transition metals, Proc. R. Soc. A 153, 699 (1936); The resistance and thermoelectric properties of the transition metals, Proc. R. Soc. A 156, 368 (1936).

[23] A. Fert and I. A. Campbell, Two-Current Conduction in Nickel, Phys. Rev. Lett. 21, 1190 (1968).

[24] P. C. van Son, H. van Kempen, and P. Wyder, Boundary Resistance of the Ferromagnetic-Nonferromagnetic Metal Interface, Phys. Rev. Lett. 58, 2271 (1987).

[25] A. Scherz, C. Sorg, M. Bernien, N. Ponpandian, K. Baberschke, H. Wende, and P. J. Jensen, Importance of giant spin fluctuations in two-dimensional magnetic trilayers, Phys. Rev. B 72, 054447 (2005).

[26] J. Li et al., Direct detection of pure spin-current by x-ray pump-probe measurements, arXiv:1505.03959. 